temperature curve ${ }^{3}$ at the same point suggest the existence of two forms of nitrobenzene.

It seemed of interest to ascertain if any change in molecular refraction is observable in the neighbourhood of this temperature. Having a supply of B.D.H. ' purified nitrobenzene' at my disposal, it was decided to carry out a fow tests with it. It melted sharply at $5 \cdot 3^{\circ} \mathrm{C}$, a temperature $0.2^{\circ}$ below that of Mazur's specimen. Its density (in air) was determined pycnometrically, with the following results :

\begin{tabular}{|r|c|c|c|}
\hline$t{ }^{\circ} \mathrm{C}$. & Density. & $t^{\circ} \mathrm{C}$. & Density. \\
\hline $6 \cdot 6$ & 1.2168 & $15 \cdot 4$ & $1 \cdot 2 \circ 82$ \\
$8 \cdot 0$ & 1.2159 & $24 \cdot 4$ & $1 \cdot 1994$ \\
11.0 & 1.2127 & $26 \cdot 1$ & $1 \cdot 1977$ \\
11.4 & 1.2122 & $29 \cdot 6$ & $1 \cdot 1943$ \\
13.4 & 1.2101 & $\ldots$ & $\cdots$ \\
\hline
\end{tabular}

The density $d$ at any temperature can be calculated with considerable accuracy from the equation, $d=1 \cdot 2233-0 \cdot 00098 t$, for, when plotted against the temperature, a rectilinear curve is obtained. The results agree very closely with those of Tyrer over his range of $13^{\circ}-30^{\circ} \mathrm{C}$., and also with the very recent data of Massy, Warren, and Wolfenden ${ }^{5}$ over their range of $6^{\circ}-14^{\circ} \mathrm{C}$. These latter data had not been published when my experiments were carried out ; in neither set of results is there any indication of discontinuity within the temperature ranges examined. The equation gives 1.2037 for the density at $20^{\circ} \mathrm{C}$., which agrees closely with 1.2039 given in the International Critical Tables.

The refractive index was determined using sodium light, with a Pulfrich refractometer, the zero of which was checked against carbon disulphide and bromo-naphthalene. The results in the accompanying table were obtained :

\begin{tabular}{|c|c|c|}
\hline Temperature ${ }^{\circ} \mathrm{C}$. & Refractive Index, $n$. & $\begin{array}{c}\text { Molecular } \\
\text { Refraction. }\end{array}$ \\
\hline $6 \cdot 4$ & 1.55972 & $56 \cdot 60$ \\
$8 \cdot 6$ & 1.55893 & $56 \cdot 62$ \\
$9 \cdot 2$ & 1.55858 & $56 \cdot 61$ \\
$10 \cdot 5$ & $1 \cdot 55786$ & $56 \cdot 60$ \\
$13 \cdot 5$ & 1.55653 & $56 \cdot 60$ \\
$15 \cdot 1$ & $1 \cdot 55565$ & $56 \cdot 59$ \\
$20 \cdot 6$ & $1 \cdot 55299$ & $56 \cdot 57$ \\
\hline
\end{tabular}

The value for $n$ at $20 \cdot 6^{\circ}$ closely approaches the value 1.55291 for $20^{\circ}$ given in the International Critical Tables (vol. 7).

The third column gives the molecular refraction calculated from Gladstone and Dale's formula, $\frac{M(n-1)}{d}$, where $M$ is the molecular weight, taken as $123 \cdot 07$, and $d$ the density as calculated with the aid of the equation given above.

The molecular refraction shows merely a minute and steady change with the temperature, amounting in all to approximately 0.1 per cent. These results, coupled with those of Massy and his co-workers, indicate that ordinarily 'pure' nitrobenzene exhibits no apparent discontinuities in such physical properties as have been examined in the neighbourhood of $9 \cdot 5^{\circ} \mathrm{C}$. To what cause, then, may the remarkable observations of Mazur be assigned?

Technical College, Birmingham,

J. Newton Friend. Feb. 4.

1 Mazur, NATURE, 126, $993 ; 1930$.

Wolfke and Mazur, NATURE, 127, $741 ; 1931$.

3 Mazur, ibid., p. 893.

- Tyrer, Trans. Chem. Soc., 105, 2544; 1914.

s Massy and others, $J$. Chem. Soc., 91; 1932.

No. 3256 , VoL. 129]

\section{Hyperfine Structure of the Mercury Line 5471 A.}

IN an attempt to explain the observed complicated structure of the mercury arc lines, Venkatesachar and Sibaiya 1,2 first suggested nuclear moments of 1/2 and $3 / 2$ for the various isotopes of mercury. More recently, Schuler and Keystone ${ }^{3}$ in Germany and Murakawa in Japan have explained the structure of some prominent mercury arc lines by ascribing a nuclear moment of $1 / 2$ to the odd isotope with atomic weight 199 and $3 / 2$ to that with 201 .

Though the mercury arc lines have been analysed by a number of investigators, a re-examination of the lines has revealed in some cases the indubitable existence of satellites that have not been previously recorded. It is realised that one has to be very cautious before being certain of the existence of new satellites, often faint, in the case of lines that have been carefully examined by others. It is concluded, however, that even the line $5461\left(6^{3} P_{2}-7{ }^{3} S_{1}\right)$ has undoubtedly one more satellite at $-0.154 \pm 0.002 \mathrm{~A}$. The structure was examined in a low density, long column mercury arc, using as the resolving apparatus fused quartz etalon plates (specially made by Messrs. Adam Hilger, Ltd.) of $2 \mathrm{~mm}$. and $3 \mathrm{~mm}$. thickness, suitably silvered on both sides. The microphotometer record (kindly taken by Messrs. Carl Zeiss) of the pattern obtained by using the $3 \mathrm{~mm}$. etalon re. produced in Fig. 1, shows clearly the position of the new satellite. Besides, the existence of a satellite in this region has been suspected by Luneland ${ }^{5}$ so early

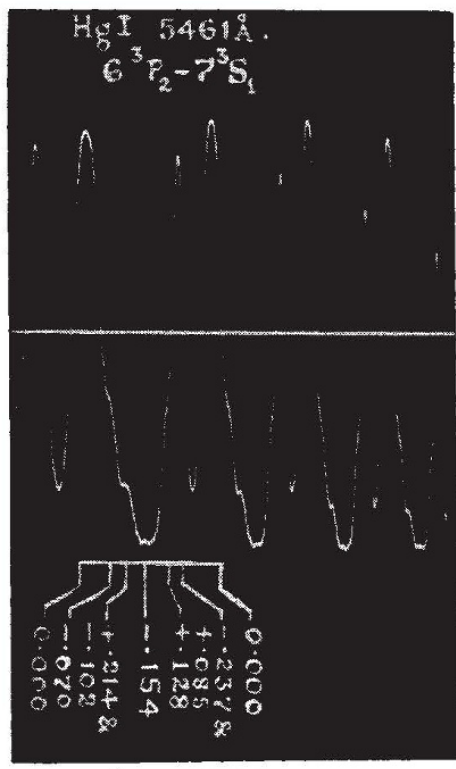

FIG. 1. as 1911, and later by Schrammen ${ }^{6}$ in 1927. But so definite a satellite does not work into the Schuler-Key stone scheme even as a forbidden satellite.

Again, the line $5770\left(6{ }^{1} P_{1}-6{ }^{3} D_{2}\right)$ has shown a structure containing three additional satellites at $-0.173,-0 \cdot 213$, and $-0.257( \pm 0 \cdot 003) \mathrm{A}$. These new satellites for 5770 along with the complex structures of $4916\left(6^{1} P_{1}-8{ }^{1} S_{0}\right)$ and $5026\left(6^{1} P_{1}-7^{3} S_{1}\right)$, recently given by Venkatesachar and Sibaiya, ${ }^{7}$ indicate that the common level $6^{1} P_{1}$ is more complicated than is assumed by Schuler and Keystone.

L. Sibaiya.

Central College,

University of Mysore,

Bangalore, Feb. 12.

${ }^{1}$ Mys. Univ. J., 4, 145-148; 1929

2 Sibaiya, "Revision of the Hyperfine Structure Data of some Prominent Mercury Lines" (abstract), 19th Ind. Sci. Cong., 1932.

3 . Phys., 72, 423-441; 1931.

- Z. Phys., 73, 366-375; 1931.

5 Ann. Physik, 34, 505; 1911.

6 Ann. Physik, 83, 1161-1199; 1927.

? Naturwiss., 19, H. 52, S. 1041-1042 ; 1931. 